\title{
Integrating the Exercise and Environmental Data Into a Digital ECG Structure by Watermarking Technique
}

\author{
Piotr Augustyniak ${ }^{1}$ \\ ${ }^{1}$ AGH University of Science and Technology, Kraków, Poland
}

\begin{abstract}
Exercise test is worldwide recognized as a valuable tool for investigating ST segment-based ischemia markers. Due to load-related risk, the test is reserved for office use, what causes inconvenience, limits patients participation rate and precludes screening for early ischemia stages. Transferring the diagnosis to patients' premises and using everyday activities as a stimulus is an interesting alternative, but needs reliable recording of physical load data. This paper presents a method for integrating the exercise and environmental data into a digital ECG structure by watermarking technique. The method analyses the time-scale ECG representation, detects the bandgap, where the bandwidth of actual cardiac content is lower than the throughput of digital series, detects the noise and replaces it by exerciserelated data. Unless in irregular signals, the capacity of data container can accommodate an accompanying accelerometer and environment-related signals without deteriorating the ECG content. This makes possible to perform ECG exercise test in home conditions without additional transmission channels or data structures. The method was tested with CSE database accordingly to EN60601-2-25:2015 and proved the watermarked ECG to maintain the wave borders accuracy within tolerance limits. Consequently, restoration of original ECG record is not necessary. The method was also tested with anonymized stress-test records, which were watermarked with accelerometer data and re-interpreted to yield results fairly comparable to original diagnoses.
\end{abstract}

\section{Introduction}

The exercise test is one of frequently used diagnostic procedures in a large population of potentially ischemic patients. The exercise is standardized by a time-load protocol (e.g. Bruce [1]) and with the usage of cycle ergometers or treadmills yields scalable quantitative results. However, attendance in repetitive tests may be cumbersome for elderly patients and thus widely observed decrease of participation rate affects the efficiency of ischemia detection. Unfortunately, due to unknown degree of circulatory deficiency, the procedure involves a risk of infarct and consequently cannot be directly applied in home care. An interesting alternative consists in using everyday activities as a load stimulus, however in this case the lack of load standardization (or protocol) requires continuous measurement of the physical exercise. This measurement is also used for realtime detection and reporting of possible load excess.

Introduction of new measurements usually requires new sensors, storage and transmission media and extension of data storage structures. This involves significant costs, poses data synchronization problems and challenges backward compatibility of new records.

In this paper we propose a cardiac-, exercise- and environmental data fusion to allow for distant modelling of patient exercise while maintaining full diagnostability of the related cardiac representation. We use an efficient irreversible watermarking technique to embed adaptively tailored data packets from additional sensors into timecritical digital electrocardiogram. The only assumption of the method is that the ECG is composed of mutually dependent band-limited components (i.e. P, QRS and T waves) separable with the software with acceptable reliability and accuracy.

\section{Data fusion concept}

Watermarking technique is usually recalled in context of data hiding, source authentication or sensitive data integration [2-4]. Each such application assumes low volume and time-independence of the watermark. Integration of the accompanying measurement results, however, requires relatively higher throughput and coding efficiency. The research reported in this paper stems from [5] and our previous projects with personal data hiding [6] and authorization-dependent local extension of ECG sampling rate [7]. The concept of the system was previously presented in [8], and successful embedment of accelerometer signals encouraged us to extend the range of measured variables by temperature, humidity and galvanic skin response.

The idea of watermarking is essentially based on a non-uniform distribution of information in host image or 
signal [9]. In case of ECG various approaches were proposed [10-11], however we use the concept of instantaneous bandwidth of the electrocardiogram (IBE) developed in our previous papers [12-13]. To determine the expected time-bandwidth relation accordingly to cardiac components in each particular heartbeat the precise delineation of ECG wave borders is followed by landmark-dependent beat-to-beat temporal scaling of the IBE. The scaling is performed in following three steps:

- stepwise resampling function is calculated as a ratio of length for each pair of corresponding sections in given heartbeat and in IBE,

- cubic splines interpolation with nodes falling in section borders is employed to determine all intermediate values of smooth resampling function,

- IBE is resampled with splines interpolation according to the local values of smooth resampling function.

Identification of time-bandwidth relation in the ongoing ECG has two purposes, both usable in a timefrequency representation of the ECG. Firstly it determines the area where cardiac components are expected and should not be affected by the watermark. Secondly it determines the area, where cardiac components are not possible to occur (i.e. difference between local ECG bandwidth and the constant value of Nyquist frequency yields a bandgap) and the host signal can accommodate watermark data instead of the intrinsic noise.

In general, steganography assumes that the watermark is invisible to a non-authorized reader. In our case the embedded exercise data are expected to be transparent to manual or machine ECG interpretation. Immunity of the diagnostic outcome to the watermarking is then dependent on how closely the watermark data mimic the noise they replace. To get best similarity, first the noise statistics (amplitude and distribution) are determined in each lead and each heartbeat independently and then the watermark data are coded with appropriate bit depth.

\section{Tables and figures}

The prototype system (fig. 1) was composed of five sensors types. Three of them are wearable:

- a three lead chest ECG (12 bits @ $500 \mathrm{~Hz})$,

- three triaxial accelerometers (12 bits@15 Hz),

- a single galvanic skin response band $(12$ bits @ $1 \mathrm{~Hz})$,

while the remaining two are stationary and provide environmental data temperature and humidity (both 12 bits @ $1 \mathrm{~Hz}$ ). All sensing nodes are working in a wireless network and controlled via Bluetooth by a dedicated mobile server. The server also run the watermarking process. For the reasons of commodity we used a laptop as the server, but all essential procedures were coded in Java in order to facilitate their future transfer to a true mobile platform (such as Android OS).

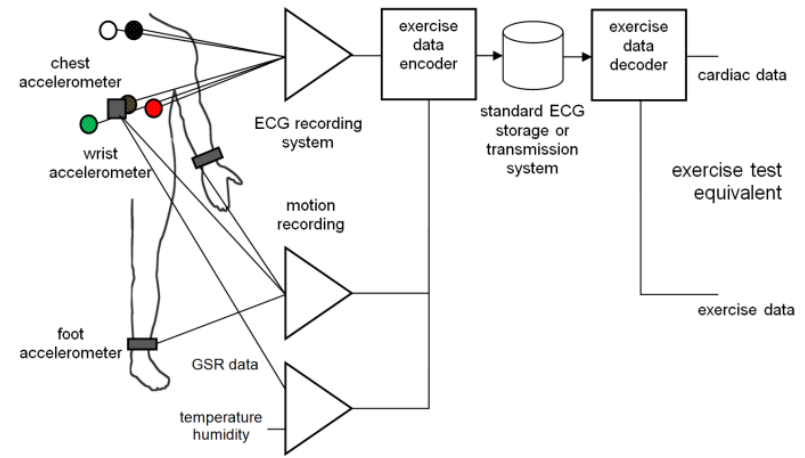

Figure 1. Block diagram of home exercise monitoring system

As a preliminary step of ECG signal processing we implemented a minimum-delay variant of Martinez algorithm [14]. This selection was motivated by high performance (sensitivity: $99.80 \%$, positive predictive value: $99.86 \%$ and false detection rate: $0.34 \%$ ) and timefrequency domain operation of the algorithm (sym11 wavelet). All watermarking procedures were performed in the time-frequency domain and at the end the temporal ECG was restored for storage and transmission in conventional 3-lead Holter recording system. The watermark keeps the exercise and ECG data properly synchronized and persists in the ECG until purposely destroyed (irreversible watermarking).

The watermarking is performed in five steps (fig. 2):

- identification of data container area in the first scale of time-scale ECG representation between the end of QRS and the beginning of $P$ waves,

- analysis of intrinsic ECG noise in the container area,

- packetizing of the exercise data and adaptive coding accordingly to the maximum noise likelihood rule,

- replacement of the intrinsic ECG noise in the container area by coded watermark data,

- coding of the data container attributes in second scale of ECG representation.

It is noteworthy that data containers are individually set accordingly to the local properties of the host ECG and noise in each heartbeat and lead. Therefore they show varying data capacity and need individual descriptions. The advantage is the resulting independence of encoded watermarks - they may contain data of different types and require autonomous authorization for access.

Since the watermarked ECG maintains all diagnostic features, it is interpreted without the need of watermarking-related processes at the recipient end. The watermark content has to be only accessed to disclose exercise test data. A decoding procedure transforms the stegano ECG to the time-scale domain, localizes and reads data container attributes and finally accesses and decodes the measurement results (fig. 3). 


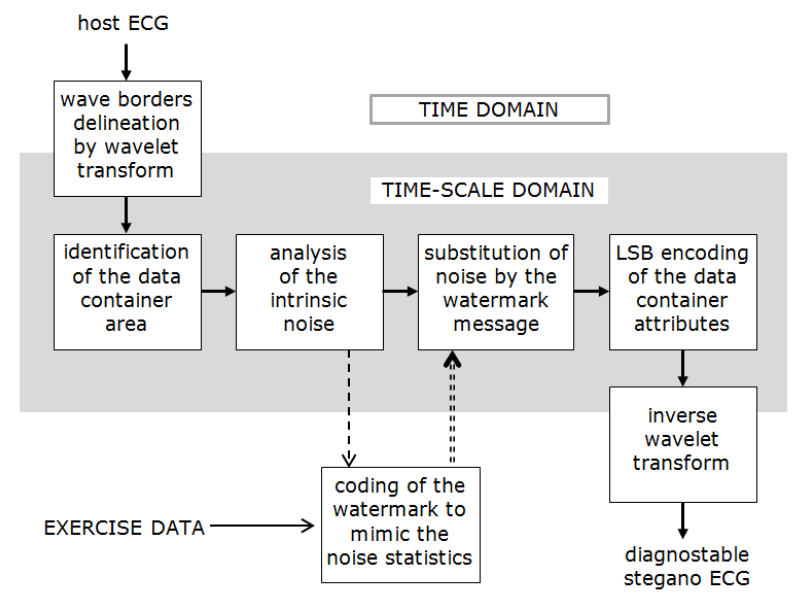

Figure 2. Time scale ECG watermarking procedure.

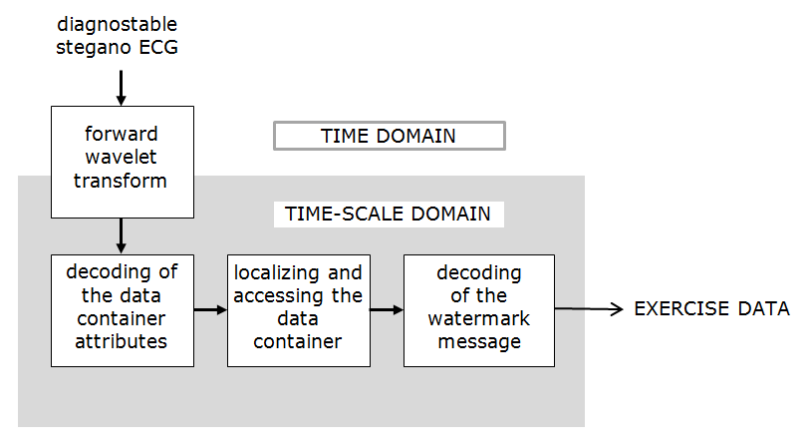

Figure 3. Time scale watermark decoding procedure.

\section{Tests and results}

In most applications of steganography the host signal or image is meaningless. Its single role is to attract the attention of the reader and to hide the existence of the watermark. The watermark is thus reproduced perfectly, while a possible alteration of the host is negligible. On the contrary in our system both the host and the watermark carry important diagnostic information, therefore first issue to test is the transparency of data fusion process. Unlike other authors evaluating the ECG watermarking with PRD or other signal metrics, we pay attention to the invariance of the diagnostic results. Consequently, with a given automatic interpretation software (Ascard 6 by Aspel, Poland) we compare the performance of clean and watermarked ECG interpretation accordingly to the EN60601-2-25:2015 procedure [15] with CSE Database [16] file subset. Results of this evaluation (tab. 1) show that only slight differences of waves delineation were caused by the watermarking and the watermarked ECG maintains all diagnostic features of the original record.
Table 1. Average results of wave delineation performance of the original ECG (a clean carrier) with its counterpart hosting exercise data [ms].

\begin{tabular}{ccccccc}
\hline \multirow{2}{*}{$\begin{array}{c}\text { ECG } \\
\text { section }\end{array}$} & \multicolumn{2}{c}{ Original } & \multicolumn{2}{c}{ Watermarked } & \multicolumn{2}{c}{ IEC Allowed } \\
& mean & std & mean & std & mean & std \\
\hline P & \pm 4.3 & 3.3 & \pm 4.5 & 3.3 & \pm 10 & 15 \\
QRS & \pm 2.1 & 1.7 & \pm 2.1 & 2.4 & \pm 10 & 10 \\
P-Q & \pm 3.8 & 4.7 & \pm 4.0 & 5.1 & \pm 10 & 10 \\
Q-T & \pm 11.3 & 7.3 & \pm 11.8 & 10.8 & \pm 25 & 30 \\
\hline
\end{tabular}

Deficient oxygenation of the heart can be detected and reliably assessed by changes in early repolarization stage expressed in measurements at the ST segment of the electrocardiogram [17]. Therefore, in case of exercise testing the accuracy of wave delineation gives priority to the precision of S-T segment measurements. In the second experiment we used original exercise test records from 17 volunteers, who performed standard exercise test (according to the Bruce protocol) with an additional accelerometer set. The clean ECG was first interpreted in real time yielding reference results. Then the ECG was watermarked with accelerometer data gathered simultaneously and interpreted again with the same software. Results of the exercise evaluation are presented in tab. 2.

Table 2. Differences of S-T segment measurement results between clean and watermarked exercise ECGs.

\begin{tabular}{lcc}
\hline \multicolumn{1}{c}{ S-T Parameter } & \multicolumn{2}{c}{ Parameter Difference } \\
& mean & std \\
\hline elevation/depression $[\mu \mathrm{V}]$ & \pm 15.7 & 41.0 \\
threshold mismatch cases & 2 & \\
slope coefficient & \pm 0.157 & 0.203 \\
slope sign mismatch cases & 1 & \\
\hline
\end{tabular}

Third issue to be addressed before transferring of the exercise test to the patients' homes is the accuracy of reproducing the physical load by the accelerometer data. This time we compare the acquired accelerometer data to the values of load known from the Bruce protocol. The results (tab. 3) were consistent for particular individuals, but show significant inter-personal differences. Moreover, the comparison was performed for a known activity (walking) well represented in motion of chest and limbs. We found that modeling of human load by the accelerometer data is not yet sufficiently studied and left it beyond the scope of this paper. Additional data on the temperature and humidity as well as galvanic skin response may also be helpful for remote load assessment. 
Table 3. Contribution of accelerometers and the particular directions in assessment of physical load during treadmill gait; $x$ stands for transverse, $y-$ for lateral and $z$ - for vertical motion.

\begin{tabular}{ccccc}
\hline \multicolumn{2}{c}{ Accelerometer } & \multicolumn{3}{c}{ axes contribution [\%] } \\
position & $\begin{array}{c}\text { contribution } \\
{[\%]}\end{array}$ & $\mathrm{x}$ & $\mathrm{y}$ & $\mathrm{z}$ \\
\hline wrist & 32 & 78 & 10 & 12 \\
chest & 16 & 12 & 8 & 80 \\
ankle & 52 & 63 & 10 & 27 \\
\hline
\end{tabular}

\section{Conclusions}

The research reported showed that time-scale domain watermarking of the ECG is efficient enough to embed data from three accelerometers and three additional sensors without noticeable degradation of wave delineation and ST parameters. This paves the way for daily homemade exercise tests as basic screening tools in preventive programs for identifying persons with possible heart disease before life-threatening symptoms occur. The diagnostic made at home is more comfortable to patients and proposed method of data fusion avoids extension of existing ECG recording and reporting infrastructure.

In home care conditions load control protocols cannot be applied, thus the physical load has to be monitored otherwise. We found accelerometers alone being not adequate to build a reliable remote representation of human load during everyday activities and therefore an optimal sensor set should be found in further research.

\section{Acknowledgments}

The scientific work is supported by the AGH University of Science and Technology in year 2018 as a research project No. 11.11.120.612.

\section{References}

[1] R. A. Bruce, "Methods of exercise testing: Step test, bicycle, treadmill, isometrics," Am. J. Cardiol. vol. 33, no. 6, pp. 715-720 1974,

[2] W. Lee, C. Lee, "A cryptographic key management solution for HIPAA privacy/security regulations," IEEE Trans. Inf. Technol. Biomed., vol. 12, no. 1, pp. 34-41, 2008

[3] H. Wang, Z. Weiming, Y. Nenghai, "Protecting patient confidential information based on ECG reversible data hiding," Multimedia Tools and Applications, vol. 75 no. 21, pp. 13733-13747, 2016

[4] C.A. Liji, K.P. Indiradevi, K.A. Babu, "Integer-to-integer wavelet transform based ECG steganography for securing patient confidential information," Procedia Technology. vol. 24, pp. 1039-1047, 2016
[5] A. Ibaida, R. van Schyndel, "A low complexity high capacity ECG signal watermark for wearable sensor-net health monitoring system," Proc. of Computing in Cardiology. vol. 38, pp. 393-396, 2011

[6] P. Augustyniak "Analysis of ECG bandwidth gap as a possible carrier for supplementary digital data," Proc. of Computing in Cardiology, vol. 39, pp. 73-76, 2012

[7] P. Augustyniak, "Encoding the electrocardiogram details in the host record's bandgap for authorization-dependent ECG quality," Proc. of Computing in Cardiology, vol. 41, pp. 465-468, 2014

[8] P. Augustyniak, "Time-frequency integration of variablebandwidth signals and supplementary data packets," International Journal of Biology and Biomedical Engineering, vol. 12, pp. 114-123, 2018

[9] N. Dey, A.S. Ashour, S. Chakraborty, S, Banerjee, E. Gospodinova, M. Gospodinov, A.E. Hassanien "Watermarking in Biomedical Signal Processing," In: N. Dey, V. Santhi, (eds.) Intelligent Techniques in Signal Processing for Multimedia Security. Studies in Computational Intelligence, vol. 660. Springer, Cham, pp. 345-369, 2017

[10] P.K. Dilip, V.B. Raskar, "Survey paper on wavelet based ECG steganography," International Journal of Research in Engineering and Technology, vol. 04 no. 03, pp. 165-169 2015

[11] J. Yoga Priya, R. Suganya, "Steganography techniques for ECG signals : A survey," Proc. of 11th International Conference on Industrial and Information Systems (ICIIS), pp. $269-273,2016$

[12] P. Augustyniak, "Pursuit of the ECG information density by data cancelling in time-frequency domain," IFMBE Proc. vol. 2, pp. 152-153, 2002

[13] P. Augustyniak, "Moving window signal concatenation for spectral analysis of ECG waves," Proc. of Computing in Cardiology, vol. 37, pp. 665-668, 2010

[14] J.P. Martínez, R. Almeida, S. Olmos, A.P. Rocha, P. Laguna, "A wavelet-based ECG delineator: evaluation on standard databases," IEEE Trans. Biomed. Eng., vol. 51, pp. 570-581, 2004

[15] European Committee for Electrotechnical Standardization (CENELEC), EN 60601-2-25:2015 Medical electrical equipment. Particular requirements for the basic safety and essential performance of electrocardiographs, 2015, ISBN 978-2-88912-719-1

[16] J.L. Willems, et al. "A reference data base for multilead electrocardiographic computer measurement programs," $J$. Am. Coll. Cardiol., vol. 10, no. 6, pp. 1313-1321 1987

[17] P.T. O'Gara, et al., "2013 ACCF/AHA guideline for the management of ST-elevation myocardial infarction," Circulation. vol.127, pp. e362-e425, 2013

Address for correspondence:

Name. Piotr Augustyniak

Full postal address. 30 Mickiewicz Ave, 30-059 Krakow, Poland

E-mail address august@agh.edu.pl 